Short Communication

\title{
Generalized ingroup-stereotyping as a response to perceived individual failure
}

\author{
Arne Roets*, Dries Bostyn, Jonas De keersmaecker, Jasper Van Assche, Alain Van Hiel \\ Ghent University, Belgium
}

A R T I C L E I N F O

\section{Keywords:}

Stereotypes

Ingroup

RWA

Performance

\begin{abstract}
A B S T R A C T
Recently, scholars have argued that people may sometimes 'strategically' endorse ingroup-stereotypes to explain their own underperformance on stereotypic tasks. In the present study $(N=453)$, after receiving bogus negative feedback on their individual performance on a math/special ability test, women showed higher agreement with statements about men being more proficient than women in these domains. This effect, however, only materialized in women who are normally less inclined to endorse stereotypes, based on their low levels of Right-Wing Authoritarianism. Moreover, also positive ingroup-stereotypes were activated simultaneously, which may further explain how ingroup-stereotyping can help protect the individual's self-esteem.
\end{abstract}

\section{Introduction}

Stereotyping has been a central topic in social psychology for decades, with a wealth of studies and theories about why people use stereotypes, and how negative stereotyping is central to prejudice and discrimination of outgroups (e.g., Lepore \& Brown, 1997). However, although considerably less prominent as a topic of interest in the literature, people may also endorse negative stereotypes about their own group (see, Bell \& Burkley, 2013). Scholars have traditionally explained negative ingroup-stereotyping as a perverse consequence of oppression and internalization of stigmatized identity (e.g., Allport, 1954; Lewin, 1948). More recently however, it has been argued that endorsement of ingroup-stereotypes can actually be 'adaptive' or 'strategic' in particular circumstances. For example, Burkley and Blanton (2008) demonstrated that endorsement of ingroup-stereotypes can be instrumental to protect oneself against threats to individual self-esteem when confronted with personal failure. The present research builds on and expands this novel line of research, testing whether the effect a) is moderated by rightwing socio-ideological attitudes, yielding stronger effects for those high in authoritarianism and b) generalizes to stereotypes beyond those directly linked to the task at hand, with simultaneous endorsement of positive in-group stereotypes regarding other domains.

\subsection{Ingroup-stereotypes as reaction to individual failure on stereotypic tasks}

Although people generally reject negative ingroup-stereotypes, some recent studies (e.g., Burkley \& Blanton, 2008, 2009; Kim, Lee, \&
Hong, 2012) have demonstrated that people may also 'temporarily' endorse such stereotypes in circumstances where they provide a viable explanation for personal underperformance. The experiments by Burkley and Blanton (2008) showed that women who received (bogus) negative feedback on their math performance (i.e., a domain stereotypically seen as 'male'), subsequently showed stronger endorsement of the 'men are better at math' stereotype. The authors argued that by putting the 'blame' for apparent underperformance on their group membership rather than on themselves, these participants could protect self-esteem in the face of failure. Indeed, women who were given an opportunity (versus no opportunity) to endorse the negative ingroupstereotypes after their apparent failure on the math task showed higher self-esteem.

However, how robust is this 'reactive' ingroup-stereotyping effect? Many studies have shown that people's general level of stereotyping is to a large degree already determined by their socio-ideological attitudes. Most relevant for gender stereotypes and benevolent sexism are social-cultural right-wing attitudes tapping into conventionalism and traditionalism (e.g., Christopher \& Mull, 2006), which are commonly operationalized using the Right-Wing Authoritarianism scale (RWA, Altemeyer, 1981). In this respect, Roets, Van Hiel, and Dhont (2012) showed that these attitudes not only strongly predict gender outgroupstereotyping, but also gender ingroup-stereotyping. Importantly, whereas individuals high in RWA by default more strongly endorse stereotypes, also low RWA individuals can exhibit high levels of stereotyping when they are 'triggered' by the context (see e.g., Van Assche, Roets, De keersmaecker, \& Van Hiel, 2017; cf. The Threat-Constraint

\footnotetext{
* Corresponding author at: Department of Developmental, Personality and Social Psychology, Henri Dunantlaan 2, B-9000 Ghent, Belgium.

E-mail address: Arne.Roets@UGent.be (A. Roets).
} 
Model by Sibley, Osborne, \& Duckitt, 2012). Therefore, we hypothesized that whereas high-RWA individuals should show endorsement of ingroup-stereotypes regardless of the situation, low-RWA individuals may be especially susceptible to the situational impact of negative stereotype task feedback on ingroup-stereotyping.

A second question pertains to the broadness of the ingroup-stereotype activation. Indeed, the intriguing research of Burkley and Blanton (2008) focused only on the effects of negative feedback on the particular stereotype that is directly relevant to the math task. However, any such specific stereotype is part of a broader cluster of stereotypes about the group: Gender stereotypes about task performance not only pertain to math, but also to other domains. Importantly, in some of these domains, such as verbal skills, and social and emotional intelligence, women are stereotypically seen as superior to men. Hence, a most relevant question is whether negative feedback solely triggers the endorsement of the negative ingroup-stereotype about the particular domain of the task, or whether the whole 'ingroup-stereotype cluster' is activated? Drawing on Social Identity Theory and stereotype-content models, Oldmeadow and Fiske (2010) demonstrated that groups may actively differentiate themselves on particular (stereotype) dimensions to pursue positive distinct identities. The potential activation of such other (i.e., positive) ingroup-stereotypes could further help us understand how 'reactive' ingroup-stereotype endorsement works, and how it may help to protect self-esteem.

\section{Method}

\subsection{Participants}

Power simulations for complex models (paramtest package ${ }^{1}$ ) showed that a sample size of 362 participants provides $80 \%$ power for detecting small-to-medium sized interaction effects $(\beta=0.15)$. We recruited a sample of 453 female undergraduate psychology students $\left(M_{\text {age }}=19.32, S D=2.94\right)$. After giving informed consent, participants completed all measures for this study as part of a larger testing session in the lab or through our online portal, and were debriefed afterwards.

\subsection{Procedure and measures}

Participants first completed the RWA scale, followed by the math and spatial tasks. After receiving bogus feedback on their math and spatial ability, they completed the gender-stereotyping measures. All self-report items were rated on 7-point Likert scales $(1=$ strongly disagree; 7 = strongly agree).

\subsubsection{Right-Wing Authoritarianism (RWA)}

An 11-item RWA scale (Altemeyer, 1981) was administered ( $\alpha=0.81, M=2.87, S D=0.62$ ). A sample item is "Obedience and respect for authority are the most important values children should learn."

\subsubsection{Math task and spatial task (bogus feedback)}

Participants were first presented with three matrices, each containing a set of 12 numbers. They were asked to indicate in each matrix which two unique numbers added up to exactly 10 (e.g., 3.81 and 6.19). Next, they received three items modelled after the Raven Progressive Matrices, in which they had to indicate which of eight complex geometric drawings completed the logical sequence. Participants were told that their overall score would be based on both accuracy and speed. Half of the participants received bogus feedback that they scored below the global average of students at the university. The other half received a message that they scored above the global average. Framing the scores as an accuracy-speed function with the global student body as a

\footnotetext{
${ }^{1}$ https://CRAN.R-project.org $/$ package $=$ paramtest.
}

reference, prevented participants to have accurate a-priori estimates of their own relative performance, which safeguards the credibility of the feedback.

\subsubsection{Gender stereotyping}

Finally, participants completed a 7-item questionnaire regarding their beliefs about gender differences in abilities. One general statement asked whether they believed that "men and women have different talents and abilities", four items stated that men are better than women when it comes to mathematical and spatial abilities, and two items stated that women are better than men when it comes to verbal and social abilities.

\section{Results}

ANCOVA analyses were conducted, including condition (positive/ negative feedback), mean centered RWA, and their interaction as independent variables, and with the stereotype agreement measures as the dependent variable. Analyses were conducted on the total stereotype agreement measure (7 items, $\alpha=0.88$ ), as well as on the measure for the math/spatial stereotype ( 4 items, $\alpha=0.83$ ) and the verbal/social stereotype ( 2 items, $\alpha=0.74$ ), separately.

Results showed that overall, gender stereotypes (total, 7-item scale) were more strongly endorsed in the negative feedback condition $\left(M=4.27, S D=1.17, \mathrm{CI}_{95}=[4.12 ; 4.42]\right)$, compared to the positive feedback condition $\left(M=4.10, S D=1.15, \mathrm{CI}_{95}=[3.95 ; 4.25]\right)$, although this general effect did not reach significance, $F(1,449)=2.63$, $\beta=0.08, p=.106$. Higher scores on RWA significantly predicted stronger endorsement of gender ability stereotypes: $F(1,449)=16.90$, $\beta=0.29, p<.001$. Most importantly, the interaction term was significant, $F(1,449)=4.93, \beta=-0.14, p=.027$, and slope analyses showed that negative (vs positive) feedback had stronger effects in women low (vs high) in RWA (see Fig. 1).

Next, to understand whether these overall effects are specific to and driven by the gender-stereotypical domain on which participants received feedback (i.e., mathematical/spatial ability), or whether these effects generalize across domains, we separately tested the impact on math/spatial gender stereotypes and verbal/social gender stereotypes.

Endorsement of the stereotype that men have superior mathematical and spatial skills was somewhat stronger in the negative feedback $\left(M=4.05, S D=1.29, \mathrm{CI}_{95}=[3.89 ; 4.21]\right)$, versus the positive feedback condition $\left(M=3.88, S D=1.25, \mathrm{CI}_{95}=[3.71 ; 4.04]\right)$, yet again this difference was not statistically significant, $F(1,449)=2.11$, $\beta=0.07, p=.147$. Higher scores on RWA significantly predicted stronger endorsement of math/spatial stereotypes: $F(1,449)=7.82$, $\beta=0.21, p=.005$. The interaction term was marginally significant; $F$ $(1,449)=3.60, \beta=-0.12, p=.058$, with slope analyses demonstrating a significant effect of the experimental condition in low-RWA, but not in high-RWA participants (see Fig. 1).

Most interestingly, a similar and even clearer pattern of results was found for endorsement of stereotypes about women being superior to men on verbal and social skills. The main effect of feedback was not significant (negative: $M=4.35, S D=1.35, \mathrm{CI}_{95}=$ [4.18; 4.53], positive: $\left.M=4.22, S D=1.38, C_{95}=[4.04 ; 4.39]\right), \quad F(1,449)=1.18$, $\beta=0.05, p=.278$. However, RWA again predicted stronger endorsement of these stereotypes: $F(1,449)=16.70, \beta=0.29, p<.001$, and the interaction term was also significant; $F(1,449)=5.60, \beta=-0.15$, $p=.018$. Slope analyses showed that the effect of negative vs positive feedback was significant for those with low levels of RWA, but absent for those high in RWA (see Fig. 1).

\section{Discussion}

The present study demonstrated that, when confronted with negative feedback about their performance on tasks in which men are stereotypically considered more proficient, female participants may 

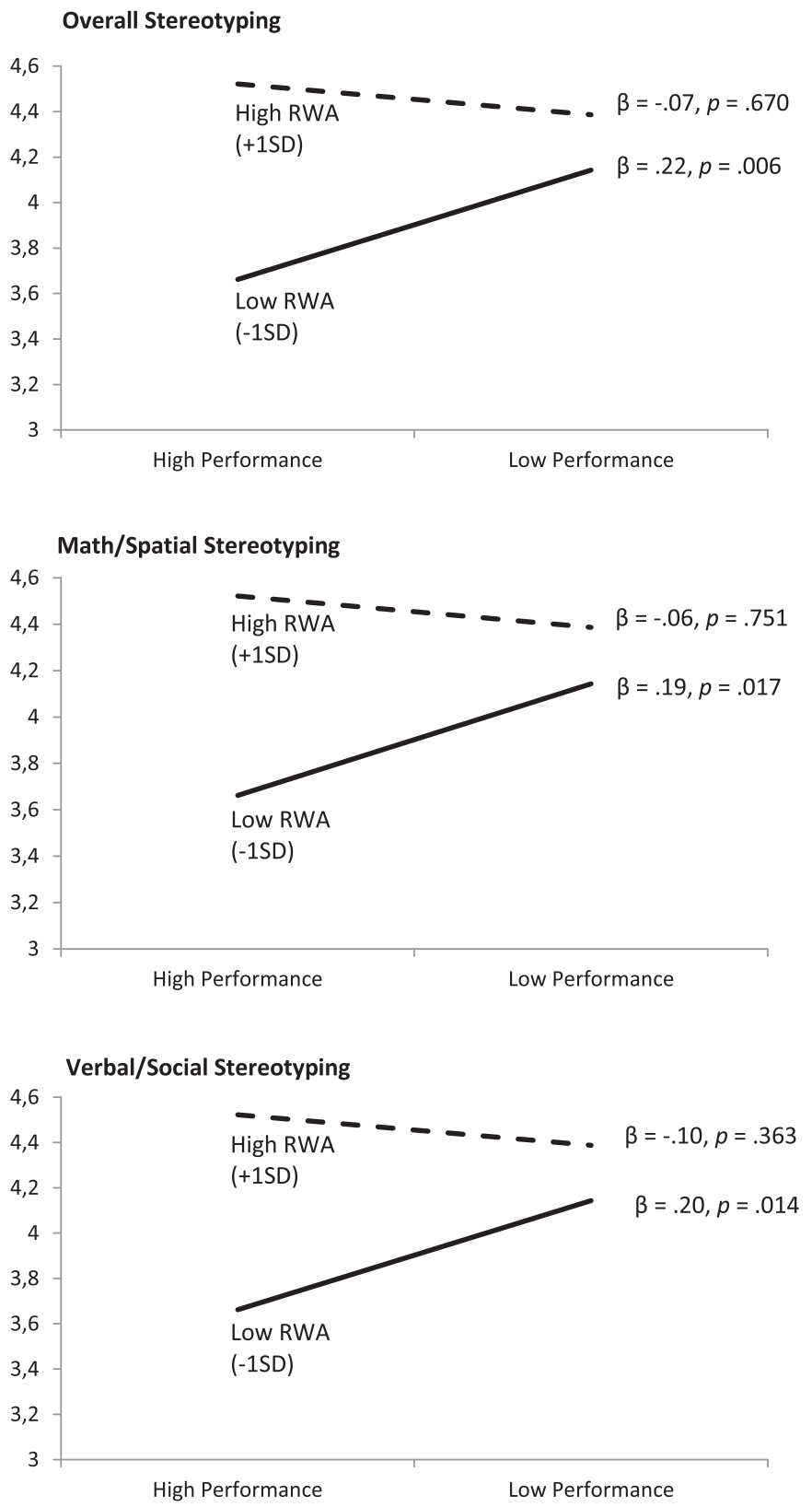

Fig. 1. Slope analyses for the interaction between RWA and performance feedback on ingroup-stereotyping.

increase their endorsement of gender stereotypes. Importantly however, this effect only occurs in women low in RWA, and in a more comprehensive way than has been described in the literature so far.

Our results fit within the recent advances in our understanding of the contextual nature of the relationship of social-cultural ideological attitudes (i.e., RWA) with stereotyping and prejudice (e.g., Van Assche et al., 2017). Indeed, although RWA is generally associated with higher levels of both outgoup- and ingroup-stereotyping (e.g., Christopher \& Mull, 2006; Roets et al., 2012), and this association can even increase under particular threats to social cohesion and structure (see e.g., Feldman \& Stenner, 1997), the present study shows that when confronted with a context that is experienced as a threat to the self (i.e., negative feedback), especially those low in RWA are most susceptible to 'reactive in-group stereotyping'.

Moreover, after receiving negative task feedback, the low-RWA participants not only concurred more with statements about men's superiority in the specific stereotypic domain of the task (i.e., math/ spatial abilities; see Burkley \& Blanton, 2008), but they also endorsed 'positive' gender ingroup-stereotypes in other domains (i.e., women's superiority for verbal and social skills). This noteworthy finding may shed a new light on the mechanisms behind the 'self-protective nature of negative ingroup-stereotyping'. Indeed, it has been argued that to protect the individual self-esteem, people may use ingroup-stereotyping to shift blame for perceived failure from the self to the group (see Burkley \& Blanton, 2008; Kim et al., 2012). The present study, however, suggests that the underlying mechanism may rather be two-fold. In particular, through activation of the "full stereotype cluster", one can attribute underperformance to stereotypic gender differences in that domain ("So I am not to blame"), while simultaneously also considering more favorable ingroup-stereotypes for other domains ("I have the potential to excel in other areas"). Future research could elaborate on this perspective and investigate to what degree self-esteem is protected by adopting the specific negative ingroup-stereotype (the 'shift blame' mechanism) and/or by the concurrent activation of positive ingroupstereotypes in other domains (the 'high potential' mechanism). Although both strategies are likely to be used simultaneously, there might be individual differences. Group-identification (see, Pickett, Bonner, \& Coleman, 2002) may be especially relevant here, as low-identifiers may be more prone to distance themselves from their group to protect their self-esteem, while high-identifiers may be more motivated to endorse the alternative, positive group-stereotypes.

Future research may also want to test these effects in male respondents. In the present study, we chose to adopt Burkley and Blanton's (2008) focus on ingroup-stereotyping in women. However, men can be expected to show the same reaction when receiving negative feedback on a task where women stereotypically are considered to perform better (e.g., on verbal, social, or emotional intelligence tasks).

\section{Conclusion}

The present study extends previous work on ingroup-stereotyping as a 'strategic means' to cope with personal underperformance in stereotypic domains. Importantly, this effect only materializes in people who are less inclined to endorse stereotypes in other circumstances (i.e., low authoritarians) and generalizes to simultaneous activation of positive ingroup-stereotypes, which may further explain how ingroup-stereotyping can help protecting self-esteem.

\section{References}

Allport, G. (1954). The nature of prejudice. Reading, MA: Addison-Wesley.

Altemeyer, B. (1981). Right-wing authoritarianism. Manitoba: University of Manitoba Press.

Bell, A. C., \& Burkley, M. (2013). "Women are bad at math": The psychological functions of negative self-stereotyping. Social and Personality Psychology Compass, 8, 708-720.

Burkley, M., \& Blanton, H. (2008). Endorsing a negative in-group stereotype as a selfprotective strategy: Sacrificing the group to save the self. Journal of Experimental Social Psychology, 44, 37-49.

Burkley, M., \& Blanton, H. (2009). The positive (and negative) consequences of endorsing negative self-stereotypes. Self and Identity, 8, 286-299.

Christopher, A. N., \& Mull, M. S. (2006). Conservative ideology and ambivalent sexism. Psychology of Women Quarterly, 30, 223-230.

Feldman, S., \& Stenner, K. (1997). Perceived threat and authoritarianism. Political Psychology, 18, 741-770.

Kim, H., Lee, K., \& Hong, Y. (2012). Claiming the validity of negative in-group stereotypes when foreseeing a challenge: A self-handicapping account. Self and Identity, 11(3), 285-303.

Lepore, L., \& Brown, R. (1997). Category and stereotype activation: Is prejudice inevitable? Journal of Personality and Social Psychology, 72, 275-287.

Lewin, K. (1948). Resolving social conflicts. New York: Harper.

Oldmeadow, J. A., \& Fiske, S. T. (2010). Social status and the pursuit of positive social identity: Systematic domains of intergroup differentiation and discrimination for high- and low-status groups. Group Processes and Intergroup Relations, 13, 425-444.

Pickett, C. L., Bonner, B. L., \& Coleman, J. M. (2002). Motivated self-stereotyping: Heightened assimilation and differentiation needs result in increased levels of positive and negative self-stereotyping. Journal of Personality and Social Psychology, 82, $543-562$.

Roets, A., Van Hiel, A., \& Dhont, K. (2012). Is sexism a gender issue? A motivated social cognition perspective on men's and women's sexist attitudes toward the own and 
other gender. European Journal of Personality, 26, 350-359.

Sibley, C. G., Osborne, D., \& Duckitt, J. (2012). Personality and political orientation: Meta-analysis and test of a threat-constraint model. Journal of Research in Personality, 46, 664-677.
Van Assche, J., Roets, A., De keersmaecker, J., \& Van Hiel, A. (2017). The mobilizing effect of right-wing ideological climates: Cross-level interaction effects on different types of outgroup attitudes. Political Psychology, 38(5), 757-776. 